LIVER

\title{
Increased prevalence of fatty liver in arterial hypertensive patients with normal liver enzymes: role of insulin resistance
}

\author{
G Donati, B Stagni, F Piscaglia, N Venturoli, A M Morselli-Labate, L Rasciti, L Bolondi
}

Gut 2004;53:1020-1023. doi: 10.1136/gut.2003.027086

See end of article for authors' affiliations

......................

Correspondence to:

Dr F Piscaglia, Department

of Internal Medicine and

Gastroenterology,

University of Bologna, via

Albertoni 15, 40138,

Bologna, Italy; Piscagl@

med.unibo.it

Accepted for publication

10 December 2003

\begin{abstract}
Background: The conditions associated with fatty liver disease presenting with normal liver enzymes and the mechanism involved in its development remain to be fully elucidated.

Aims: The aim of the present study was to test the hypothesis that fatty liver with normal liver enzymes occurs more frequently in arterial hypertensive patients and to establish whether this condition is associated with insulin resistance.

Patients: A total of 55 non-obese, non-diabetic, non-heavy alcohol drinking patients with arterial hypertensive and normal liver enzymes and 55 sex and age matched healthy subjects were enrolled into the study.

Methods: Plasma metabolic parameters, body mass index, and the presence of fatty liver were investigated. Insulin resistance was estimated from plasma insulin and glucose as the homeostasis model assessment index. Stepwise logistic regression and multivariate regression analysis were used on the combined sample to identify variables independently associated with fatty liver and insulin resistance.

Results: Hypertensive patients had a significantly higher prevalence of fatty liver $(30.9 \%$ v $12.7 \%$; $p<0.041$ ), higher insulin resistance (mean 2.27 (SD 1.81) v $1.56(0.70) ; p=0.022$ ), and slightly higher body mass index $(24.9$ (3.0) $\vee 24.0(2.2) ; p=0.043)$ than controls. Multivariate logistic regression identified insulin resistance (odds ratio 1.66 (95\% confidence interval (CI) 1.03-2.52)) and body mass index (OR 1.22 (95\% Cl 1.00-1.49)) as factors independently associated with fatty liver. Multivariate regression analysis showed insulin resistance to be predicted by alanine transaminase $(p=0.002)$, presence of arterial hypertension $(p=0.029)$, and body mass index $(p=0.048)$.

Conclusion: The higher prevalence of non-alcoholic fatty liver in non-obese hypertensive patients with normal liver enzymes appears to be related to increases in insulin resistance and body weight.
\end{abstract}

5 i atty liver most often occurs in subjects with normal levels of plasma liver enzymes, as appears to be the case in approximately $10-15 \%$ of the general population. ${ }^{12}$ As the risk of progression of fatty liver to more severe liver disease is considered negligible under these circumstances, ${ }^{3-5}$ no further diagnostic assessment is recommended, apart from periodic assay of liver enzymes to confirm levels remain normal. ${ }^{1}$ However, the real clinical meaning of fatty liver disease, with normal liver enzymes, and the mechanism involved in its development remain to be elucidated. This is particularly true when even the most common conditions associated with an increased prevalence of fatty liver are excluded-namely, obesity, ${ }^{2}{ }^{6}$ high alcohol intake, ${ }^{2}$ diabetes, ${ }^{78}$ and chronic viral hepatitis, particularly of the $\mathrm{C}$ type. ${ }^{10}$

Bearing in the mind the mechanisms involved in nonalcoholic steatohepatitis, ${ }^{11-15}$ we worked on the hypothesis that the presence of only the first hit (namely, insulin resistance) was involved in the development of fatty liver with no elevation in transaminases.

Interestingly, essential hypertension is considered an insulin resistant state $^{16}$ and approximately $50 \%$ of patients with arterial hypertension are reported to be insulin resistant with hyperinsulinaemia, ${ }^{17}$ a value much higher than that found in the general population. These metabolic abnormalities also seem to be involved in the pathogenesis of the complications of hypertension, ${ }^{18-20}$ both in obese and nonobese patients. These circulatory and metabolic disturbances can also be found in normotensive first degree relatives of hypertensive patients.

Briefly, it is tempting to speculate that fatty liver, if indeed related to insulin resistance, would occur more frequently in hypertensive patients. This hypothesis however has not been ascertained in subjects free from the above mentioned risk factors for fatty liver-namely, obesity, chronic hepatitis, alcohol abuse, and diabetes.

The aim of the present study therefore was to test this hypothesis-that is, that fatty liver with normal liver enzymes occurs more frequently in arterial hypertensive patients as it is associated with insulin resistance.

\section{PATIENTS AND METHODS}

\section{Study population}

From March 1999 to February 2001, all hypertensive patients attending the Hypertension Unit of one Division of Internal Medicine at the Department of Internal Medicine and Gastroenterology, Bologna University, were screened for increased serum transaminases, gamma glutamyl transpeptidase $(\gamma \mathrm{GT})$, as well as aetiological factors of chronic liver disease. The latter also included careful recording of patient history (with estimation of alcohol intake) and laboratory tests for hepatitis B and C virus infection. The diagnosis of hypertension in these patients was based on the following criteria: systolic blood pressure $\geqslant 140 \mathrm{~mm} \mathrm{Hg}$ and/or diastolic blood pressure $\geqslant 90 \mathrm{~mm} \mathrm{Hg}$ (measured three times within 30 minutes in the sitting position using a brachial sphygmomanometer) or ongoing antihypertensive pharmacological treatment after a diagnosis of arterial hypertension. The following were considered exclusion criteria in order not to

Abbreviations: $\gamma \mathrm{GT}$, gamma glutamyl transpeptidase; US, ultrasonography; $\mathrm{HDL}$, high density lipoprotein; ALT, alanine transaminase; AST, aspartate transaminase; HOMA, homeostasis model assessment index 
include accepted conditions considered risk factors for fatty liver: alcohol consumption $>20 \mathrm{~g} /$ day, body mass index $>30 \mathrm{~kg} / \mathrm{m}^{2}$, diabetes (fasting glucose levels $>126 \mathrm{mg} / \mathrm{dl}$ in two consecutive samples), secondary hypertension, increased transaminases, or $\gamma \mathrm{GT}$, hepatitis B or $\mathrm{C}$ infection, or other known causes of liver disease.

Patients taking drugs known to cause hepatic fat deposition (that is, corticosteroids, tricyclic antidepressants, synthetic oestrogens, chronic salicylates, tetracyclines, and amiodarone) $)^{12122}$ were also excluded. Cigarette smoking was not considered a criterion for exclusion.

Of the 259 hypertensive patients assessed, 55 (26 males, 29 females; mean age 52.7 (SD 10.0) years) fulfilled the inclusion criteria and were enrolled into the study.

A group of 55 sex and age matched ( \pm 3 years) normotensive subjects, selected according to the same exclusion criteria, comprised the control group. All had been seen as outpatients and were selected from those who sought consultation (for headache, dyspepsia, tiredness, anxiety, constipation, arthralgia, dizziness, fainting, and cough) and in whom any significant organic disease had been definitively ruled out. All individuals gave oral informed consent to take part in the study, the protocol of which conformed to the guidelines of the Declaration of Helsinki and was approved by the department ethics committee.

\section{Methods}

Blood tests and abdominal ultrasonography (US) were performed in all individuals.

Fasting serum glucose, insulin, total and high density lipoprotein (HDL) cholesterol, triglycerides, $\gamma \mathrm{GT}$, and transaminases (alanine (ALT) and aspartate (AST) transaminase) were evaluated by standardised routine laboratory techniques. Reference range of values, in our laboratory, are 0-40 IU/l for ALT, 0-37 IU/l for AST, 6-20 $\mu \mathrm{IU} / \mathrm{ml}$ for insulinaemia, 0-50 IU/l for $\gamma \mathrm{GT}, 70-170 \mathrm{mg} / \mathrm{dl}$ for triglycerides, $60-110 \mathrm{mg} / \mathrm{dl}$ for glucose, and up to $200 \mathrm{mg} / \mathrm{dl}$ for total cholesterol. The index of insulin resistance was calculated according to the homeostasis model assessment index $(\mathrm{HOMA})$ as insulin resistance $=$ insulin in $\mu \mathrm{IU} / \mathrm{ml} \times$ glucose in $\mathrm{mmol} / \mathrm{l} / 22.5 .^{23}$

It has been demonstrated that HOMA closely correlates with the insulin resistance index measured by euglycaemic clamp, even though it has a suboptimal reproducibility, reflecting day to day variability in fasting glucose and particularly insulin levels. ${ }^{23}$ Height and weight were measured, in light clothing and without shoes, at the time of the clinical examination on entry into the study.

The upper abdomen, including specifically the liver, was always assessed at US by the same experienced operator (NV) unaware of the laboratory findings and study group of the subjects under investigation. US was carried out using a 3.5 MHz convex transducer (Esaote-Hitachi AU 590 Asynchronous, Tokyo, Japan) with the patient having fasted for at least five hours. The diagnosis of fatty liver was based on the US finding of "bright" liver tissue with fine tightly packed echoes, making it significantly more echoic than the adjacent right kidney. ${ }^{24}$

\section{Statistical analysis}

Results are expressed as mean (SD) and frequencies. Differences between the two groups were tested by means of the $t$ test for paired data and the McNemar test. Intragroup analysis, according to the presence of fatty liver, was performed using the $t$ test for unpaired data. SSPS software (SPSS PC, version 8.0, Chicago, Illinois, USA) was used for statistical analysis. In order to identify variables independently associated with fatty liver, a forward stepwise logistic regression was performed on the combined samples of 110 individuals, taking into account the hypertensive status, in addition to other metabolic parameters, as independent variables. The odds ratio (OR) together with the 95\% confidence interval (95\% CI), evaluated by logistic regression, are also reported. Associations of insulin resistance with age, hypertensive status, body mass index, ALT, cholesterol (total and HDL), and triglycerides were tested by forward stepwise multivariate regression analysis. Liver status was not included in this analysis as its relationship with insulin resistance had already been evaluated by logistic regression.

\section{RESULTS}

Hypertensive patients showed a significantly higher prevalence of fatty liver (17/55 patients, $30.9 \%)$ than control subjects $(7 / 55,12.7 \%$; $=0.041)$.

Insulin levels and insulin resistance were significantly higher in the hypertensive group (table 1) whereas body mass index only approached statistical significance (table 1). Total cholesterol showed a trend to higher values in hypertensive patients (table 1). The remaining variables were not significantly different between the two groups. The mean differences between the two groups were 0.72 (95\% CI 0.17 / 1.26) for insulin resistance and $2.55(0.60 / 4.49) \mu \mathrm{IU} / \mathrm{ml}$ for plasma insulin whereas the same parameters for body mass index and glucose, variables only close to statistical significance were, respectively $0.91(-0.08 / 1.90) \mathrm{kg} / \mathrm{m}^{2}$ and 4.42 $(-0.26 / 9.10) \mathrm{mg} / \mathrm{l}$.

Intragroup analysis showed glucose and insulin resistance (fig l) to be higher in hypertensive patients with fatty liver $(\mathrm{n}=17)$ than in those with a normal liver $(\mathrm{n}=38)$ (plasma glucose 99.2 (11.4) $v 88.6$ (10.4) mg/l, respectively; $<<0.001$ ).

Table 1 Comparison of biochemical and demographic parameters between hypertensive patients and control subjects

\begin{tabular}{lccl} 
& Hypertensives $(\mathbf{n = 5 5 )}$ & Controls $(\mathbf{n = 5 5 )}$ & $\mathbf{p}$ Value \\
\hline Fasting serum insulin $(\mu \mathrm{lU} / \mathrm{ml})$ & $9.67(6.50)$ & $7.13(2.66)$ & 0.011 \\
Fasting serum glucose $(\mathrm{mg} / \mathrm{l})$ & $91.9(11.7)$ & $87.5(10.1)$ & 0.064 \\
Insulin resistance $(\%)$ & $2.27(1.81)$ & $1.56(0.70)$ & 0.011 \\
Body mass index $\left(\mathrm{kg} / \mathrm{m}^{2}\right)$ & $24.9(3.0)$ & $24.0(2.2)$ & 0.073 \\
Serum total cholesterol $(\mathrm{mg} / \mathrm{dl})$ & $207.3(31.0)$ & $195.4(41.1)$ & 0.077 \\
Serum HDL cholesterol $(\mathrm{mg} / \mathrm{dl})$ & $48.8(11.5)$ & $48.8(11.7)$ & 0.983 \\
Serum triglycerides $(\mathrm{mg} / \mathrm{dl})$ & $110.6(55.3)$ & $107.4(51.1)$ & 0.619 \\
AST (IU/l) & $19.9(4.9)$ & $18.9(4.2)$ & 0.232 \\
ALT (IU/l) & $22.0(8.2)$ & $20.4(7.5)$ & 0.222 \\
$\gamma$ GT (IU/l) & $23.6(13.6)$ & $25.5(19.3)$ & 0.545 \\
Age (years) & $52.7(10.0)$ & $52.5(10.0)$ & 0.219 \\
\hline
\end{tabular}

Data are mean (SD); $\mathrm{p}$ values refer to $t$ test for paired data.

$\mathrm{HDL}$, high density lipoprotein; AST, aspartate transaminase; ALT, alanine transaminase; $\gamma \mathrm{GT}$, gamma glutamyl transpeptidase. Insulin resistance was estimated as the homeostasis model assessment index (see methods). Upper reference limits are $37 \mathrm{IU} / \mathrm{I}$ for AST, $40 \mathrm{IU} / \mathrm{I}$ for ALT, and $50 \mathrm{IU} / \mathrm{I}$ for $\gamma \mathrm{GT}$, in our laboratory. 


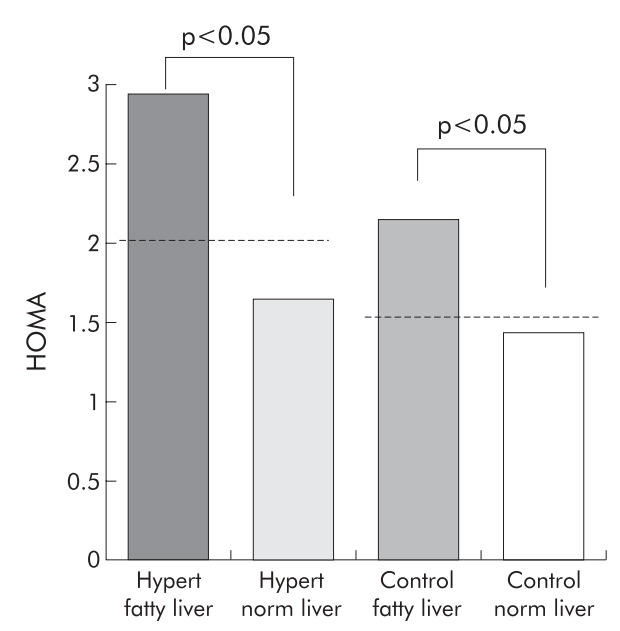

Figure 1 Homeostasis model assessment index (HOMA), an indicator of insulin resistance. Values are mean (SD) in hypertensive (Hypert) patients and control subjects subgrouped according to presence or absence of fatty liver. $p$ values indicate significance level of differences between subjects with and without fatty liver in hypertensive patients and in controls, as calculated using the unpaired $t$ test.

Body mass index was also significantly higher $(26.4(2.6) v$ $24.3(3.2) \mathrm{kg} / \mathrm{m}^{2}$, respectively; $\mathrm{p}=0.020$ ) whereas the difference in ALT and plasma insulin between hypertensive patients with and without fatty liver did not reach statistical significance $\left(\begin{array}{lllll}24.3 & (9.3) & v & 20.8 & (7.5)\end{array}\right) \mathrm{IU} / \mathrm{l}$, respectively $(\mathrm{p}=0.103)$ for ALT; and $11.9(6.0) \vee 8.7(6.5) \mu \mathrm{IU} / \mathrm{ml}$, respectively $(p=0.090)$ for insulin). The remaining parameters did not differ, although HDL cholesterol showed a clear trend to lower values and triglycerides to higher values in those with fatty liver (approximately $\mathrm{p}=0.07$ in both).

Insulin resistance (fig 1) and fasting serum insulin (9.6 (4.1) $v 6.8(2.2)$, respectively; $\mathrm{p}=0.008)$ were higher in healthy controls with fatty liver $(n=7)$ in comparison with those with a normal liver $(n=48)$. ALT was also significantly higher in controls with fatty liver $(26.6$ (6.6) $v 19.5$ (7.1) IU/l; $\mathrm{p}=0.017$ ) despite being within the normal range in all subjects. Interestingly, body mass index was not significantly different in controls with fatty liver compared with those without $\left(\begin{array}{lllll}24.7 & (1.9) & v & 23.9 & (2.3) \mathrm{kg} / \mathrm{m}^{2}\end{array}\right.$, respectively; $\mathrm{p}=0.418)$. Glucose was also higher in controls with fatty liver (96.6 (12.6) $v 86.1(9.1) \mathrm{mg} / \mathrm{l} ; \mathrm{p}=0.010)$ whereas all other parameters were clearly not significantly different.

Logistic regression selected insulin resistance (OR 1.61 (95\% CI 1.03-2.52); $\mathrm{p}=0.037$ ) and body mass index (OR 1.22 (95\% CI 1.00-1.49); $\mathrm{p}=0.048$ ) as factors independently associated with fatty liver. Moreover, ALT $(p<0.001)$, hypertension $(p=0.029)$, and body mass index $(p=0.047)$ were found to be positively associated with insulin resistance in multivariate regression analysis.

\section{DISCUSSION}

The present study, assessing the presence of non-alcoholic fatty liver disease in non-obese non-diabetic subjects with normal liver enzymes, demonstrated an increased prevalence of this hepatic abnormality in patients with primary hypertension and found it to be positively associated with insulin resistance and body weight.

The prevalence of fatty liver in our series of hypertensive patients was more than twofold that in the control group. In the latter, it was similar to that found in non-obese, nondiabetic, non-heavy drinking subjects enrolled in an epidemiological study performed in a region close to ours $(16.4 \%$ in the study by Bellentani et al $v 12.9 \%$ in the present study), ${ }^{2}$ in keeping with our results. In addition to a greater prevalence of fatty liver, our hypertensive group showed many features of the metabolic syndrome-namely, higher insulin, glucose, and insulin resistance, and a clear trend towards higher cholesterol which, according to the literature, are associated with arterial hypertension in approximately 50\% of patients. ${ }^{17} 19$

Regression analyses suggest that the association between hypertension and fatty liver is mediated by elevated insulin resistance. In fact, arterial hypertension was associated with insulin resistance in the multivariate analysis and, in turn, fatty liver was associated with insulin resistance but not with hypertension, in the logistic regression. It also appears that body weight plays a role in determining fatty liver, independent of insulin resistance. The independent role of the two conditions, shown by logistic regression, is further supported by the finding that healthy controls with fatty liver had a clear increase in insulin resistance, despite body mass index not being significantly different between the two groups.

In this study, no liver biopsy was performed as no invasive procedures were permitted by the ethics committee due to the possible complications associated with this procedure and the fact that the subjects enrolled had no biochemical signs of relevant active liver disease (that is, increase in liver enzymes or hepatitis viruses). This is in agreement with the clinical practice of the large majority of hepatologists in USA $^{1}$ who do not recommend biopsy in patients with fatty liver and normal liver enzymes. ${ }^{1}$ However, Joseph et al and Saverymuttu and colleagues $^{24}{ }^{25}$ who performed both US scans and liver biopsy with histological examination in previous studies, found a notable agreement between the two techniques in the diagnosis of fatty liver. Therefore, the diagnosis of nonalcoholic fatty liver disease was based on US examination and on exclusion of alcohol abuse and other known aetiological factors of liver disease, as generally accepted in the literature for this condition. ${ }^{26}$

We used liver transaminases as a surrogate marker of fatty liver without relevant hepatitis. A surrogate marker was needed as liver biopsy was impracticable in our protocol on account of the risk-benefit balance, as discussed above. However, the present results, which show a strong association between insulin resistance and transaminases (despite the latter being within the normal range), suggest that insulin resistance may play a role not only in hepatic lipid accumulation but also in liver inflammation and necrosis, in accordance with the pathogenesis of non-alcoholic steatohepatitis. ${ }^{11}{ }^{12}$ It is also tempting to speculate that patients with fatty liver and liver enzymes in the upper range might already be affected by mild non-alcoholic steatohepatitis. This finding would justify liver biopsy in such subjects in future studies. These results are in keeping with a recent study ${ }^{27}$ in which liver transaminases were reported to be strongly related to insulin sensitivity in Pima Indians, so that the higher the transaminases (also at abnormal values), the lower the insulin sensitivity, independent of body weight. When patients were followed up prospectively, elevated ALT was a good predictor of the future development of type 2 diabetes. $^{27}$

In the light of the data emerging from the present investigation, the question arises of whether healthy subjects with an incidental finding of fatty liver should be considered more prone to developing arterial hypertension and hence might benefit from more frequent monitoring of arterial pressure. The answer would require a long term prospective assessment. However, the association between fatty liver and insulin resistance shown herein, considered in the light of previous results reporting a role of glucose homeostasis disturbance in predicting subsequent development of hypertension, ${ }^{28}$ seems 
to support this speculation. If this is confirmed, the presence of fatty liver, without elevation of transaminases, could no longer be considered just the hallmark of a gourmet, at least in connection with cardiovascular disease.

In conclusion, our data indicate that the presence of fatty liver with normal liver enzymes is also positively associated with insulin resistance and body weight in non-obese nondiabetic subjects, and that it occurs more frequently in hypertensive patients.

\section{ACKNOWLEDGMENTS}

We are grateful to Mrs Marian Shields for assistance with the English writing of this paper.

\section{Authors' affiliations}

G Donati, B Stagni, F Piscaglia, A M Morselli-Labate, L Rasciti,

L Bolondi, Division of Internal Medicine, Department of Internal Medicine and Gastroenterology, University of Bologna, Azienda S Orsola-Malpighi, Bologna, Italy

N Venturoli, Regional Center for Transplantation, Azienda S OrsolaMalpighi, Bologna, Italy

\section{REFERENCES}

1 Younossi Z, Diehl A, Ong J. Non alcoholic fatty liver disease: an agenda for clinical research. Hepatology 2002;35:746-52.

2 Bellentani S, Saccoccio G, Masutti F, et al. Prevalence of and risk factors for hepatic steatosis in Northern Italy. Ann Intern Med 2000;132:112-17.

3 Falck-Ytter Y, Younossi Z, Marchesini G, et al. Clinical features and natural history of non alcoholic steatosis syndromes. Semin Liver Dis 2001;21:17-26.

4 Teli M, James $O$, Burt A, et al. The natural history of nonalcoholic fatty liver: a follow-up study. Hepatology 1995;22:1714-19.

5 Powell E, Cookesley W, Hanson R, et al. The natural history of nonalcoholic steatohepatitis: a follow-up study of forty-two patients for up to 21 years. Hepatology 1990;11:74-80.

6 Braillon A, Capron J, Herve M, et al. Liver in obesity. Gut 1985;26:133-9.

7 James O, Day P. Nonalcoholic steatohepatitis (NASH): a disease of emerging identity and importance. J Hepatol 1998;29:495-501.

8 Kemmer N, McKinney K, Xiao S, et al. High prevalence of non alcoholic steatohepatitis among Mexican females with type II diabetes mellitus. Gastro 2001;120:A117.

9 Czaja A, Carpenter H, Santrach P, et al. Host- and disease-specific factors affecting steatosis in chronic hepatitis C. J Hepatol 1998;29:198-206.
10 Rubbia-Brandt L, Quadri R, Abid K, et al. Hepatocyte steatosis is a cytopathic effect of hepatitis C virus genotype 3. J Hepatol 2000;33:106-15.

11 Sanyal J, Campbell-Sargent C, Mirshahi F, et al. Nonalcoholic steatohepatitis: association of insulin resistance and mitochondrial abnormalities. Gastro 2001;120:1138-92.

12 Marchesini G, Brizi M, Morselli-Labate A, et al. Association of nonalcoholic fatty liver disease with insulin resistance. Am J Med 1999; 107:450-5.

13 Lee J, Rhee $\mathrm{P}$, Lee J, et al. Role of hyperinsulinemia and glucose intolerance in the pathogenesis of nonalcoholic fatty liver in patients with normal body weight. Korean J Intern Med 1998;13:12-14.

14 Marchesini G, Brizi M, Bianchi G, et al. Metformin in non-alcoholic steatohepatitis. Lancet $2001 ; 358: 893-4$.

15 Fong D, Nehra V, Lindor K, et al. Metabolic and nutritional considerations in nonalcoholic fatty liver. Hepatology 2000;32:3-10.

16 Ferrannini E, Buzzigoli G, Bonadonna R, et al. Insulin resistance in essential hypertension. N Engl J Med 1987;317:350-7.

17 Pollare T, Lithell H, Berne C. Insulin resistance is a characteristic feature of primary hypertension independent of obesity. Metabolism 1990;39:167-74

18 Laakso M, Edelman S, Brechtel G, et al. Decreased effect of insulin to stimulate skeletal muscle blood flow in obese man. A novel mechanism for insulin resistance. J Clin Invest 1990;85:1844-52.

19 Landsberg L. Insulin resistance and hypertension. Clin Exp Hypertens 1999;21:885-94.

20 Steinberg $\mathbf{H}$, Tarshoby $M$, Monestel $R$, et al. Elevated circulating free fatty acid levels impair endothelium-dependent vasodilatation. J Clin Invest 1997; 100:1230-9.

21 Berson A, Beco VD, Letteron P, et al. Steatohepatitis-inducing drugs cause mitochondrial dysfunction and lipid peroxidation in rat hepatocytes. Gastro 1998; 114:764-74.

22 Fromenty B, Pessayre D. Inhibition of mitochondrial beta-oxidation as a mechanism of hepatotoxicity. Pharmacol Ther 1995;67:101-54.

23 Matthews D, Hosker J, Rudenski A, et al. Homeostasis model assessment insulin resistance and beta-cell function from fasting plasma glucose and insulin concentrations in man. Diabetologia 1985;28:412-19.

24 Saverymuttu S, Joseph A, Maxwell J. Ultrasound scanning in the detection of hepatic fibrosis and steatosis. BMJ 1986;292:13-15.

25 Joseph A, Saverymuttu S, Al-Sam S, et al. Comparison of liver histology with ultrasonography in assessing diffuse parenchymal liver disease. Clinical Radiol 1991;43:26-31.

26 Franzese A, Vajro P, Argenziano A, et al. Liver involvement in obese children: ultrasonography and liver enzyme levels at diagnosis and during follow up in an Italian population. Dig Dis Sci 1997;42:1428-32.

27 Vozarova B, Stefan N, Lindsay R, et al. High alanine aminotransferases is associated with decreased hepatic insulin sensitivity and predicts the development of type 2 diabetes. Diabetes 2002;51:1889-95.

28 Kashiwabara $\mathbf{H}$, Inaba $\mathrm{M}$, Maruno $\mathrm{Y}$, et al. Insulin levels during fasting and the glucose tolerance test and Homa's index predict subsequent development of hypertension. J Hypertens 2000;18:83-8. 